\title{
Exponentially practical stability of impulsive discrete time system with delay
}

\author{
Sirilak Wangrat and Piyapong Niamsup*
}

\section{"Correspondence:}

piyapong.n@cmu.ac.th

Department of Mathematics,

Chiang Mai University, Chiang Mai,

50200, Thailand

\begin{abstract}
In this paper we investigate an impulsive discrete time system with delay. By using Lyapunov stability theory and a Razumikhin type technique, some new criteria for the exponentially practical stability of impulsive discrete time system with delay are established. A numerical example is given to show the effectiveness of our theoretical results.
\end{abstract}

Keywords: discrete time system; delay; impulse; exponentially practical stability

\section{Introduction}

According to a study, a discrete time system is a more natural way to represent systems such as networked control system, numerical analysis, and population models [1-9]. In most physical dynamic systems, abrupt state changes at certain moments often occur. It is natural to assume these changes to happen instantaneously. Such dynamical systems with these changes are called impulsive systems. Impulsive systems arise in many fields and there are several studies on stability of impulsive systems [3-5, 8, 10-15]. Moreover, the real processes in our world always involve time delay systems; for example, manufacturing processes, digital control systems, population dynamics, and the electronic implementation of analog neural networks, etc. It is well known that time delay might cause instability, divergence behavior, and oscillation of dynamic systems. Therefore, the stability of impulsive system with time delay has been investigated extensively over the past decades [1-22].

Recently, the stability analysis of time delay system has been investigated extensively in many areas $[1-4,6,8,9,13,23]$. There are important types of stability of dynamical systems, namely exponential stability and practical stability. In the case of exponential stability, it is required that all solutions starting near an equilibrium point not only stay nearby, but tend to the equilibrium point very fast with exponential decay rate; see $[2-4,6,12,14,15,23]$. Meanwhile, for practical stability, one only needs to stabilize a system in a region of phase space, namely the system may oscillate close to the state, in which the performance is still acceptable. There are several results on the practical stability for continuous time systems with delay $[10,16,17]$ and without delay [12, 24, 25]. On the other hand, there are few results on practical stability for discrete time systems with delay [5, 7]. Moreover, there are several practical applications of impulsive discrete time system with delay such as BAM neural networks [18], Nicholson's blowflies model [3], and switched systems with delays [19-21]. Therefore, it is important to investigate

(c) 2016 Wangrat and Niamsup. This article is distributed under the terms of the Creative Commons Attribution 4.0 International License (http://creativecommons.org/licenses/by/4.0/), which permits unrestricted use, distribution, and reproduction in any medium, provided you give appropriate credit to the original author(s) and the source, provide a link to the Creative Commons license, and indicate if changes were made. 
the practical stability problem of impulsive discrete time system with delay. In [3, 4], the authors have studied discrete time delayed dynamic systems with impulsive effects. By employing Lyapunov stability theory and a Razumikhin type technique, exponential stability criteria of impulsive discrete time system with delay have been derived. In [5], an asymptotically practical stability condition of impulsive discrete systems with time delays has been provided by using Lyapunov stability theory and a Razumikhin type technique. However, the stability criteria depend on $\tau$, namely, $\tau \triangleq \sup _{m \in \mathbb{Z}^{+}}\left\{k_{m+1}-k_{m}\right\}<+\infty$, where $k_{m}$ are impulsive moments. Obviously, exponential stability implies exponentially practical stability but not conversely. However, in several practical applications, one only needs to stabilize a system in a region of phase space, namely the system may oscillate near the equilibrium point, in which the performance is still acceptable. Motivated by the above discussions, in this paper, we derive novel exponentially practical stability criteria of impulsive discrete time systems with delay by using Lyapunov stability theory and a Razumikhin type technique. To the best of our knowledge, it is the first result on the exponentially practical stability of impulsive discrete time systems with delay. Moreover, comparing to [5] which proposed an asymptotically practical stability condition, our technique can be used to derive an exponentially practical stability condition if it is more desirable. Furthermore, the obtained condition is not required that $\sup _{m \in \mathbb{Z}^{+}}\left\{k_{m+1}-k_{m}\right\}<+\infty$, where $k_{m}$ are impulsive moments which is imposed in [5]. The paper is organized as follows. In Section 2, we introduce some notations, definitions, and a proposition. In Section 3, we give new criteria for exponentially practical stability of impulsive discrete time systems with delay. In Section 4, a numerical example is given to show the effectiveness of our theoretical results. Our conclusion is given in Section 5 .

\section{Preliminaries}

Let $\mathbb{R}^{n}$ denote the $n$ dimensional Euclidean space, $\|x\|$ is the Euclidean norm of vector $x$. Given a positive integer $\tau$, for any function $\phi: \mathbb{N}_{-\tau} \longrightarrow \mathbb{R}^{n}$, we define $\|\phi\|=$ $\max _{\theta \in \mathbb{N}_{-\tau}}\{\|\phi(\theta)\|\}, \mathbb{N}=\{0,1,2, \ldots\}$ and $\mathbb{N}_{-\tau}=\{-\tau,-\tau-1, \ldots,-1,0\}$.

Consider the following impulsive discrete time system with delay:

$$
\left\{\begin{array}{l}
x(k+1)=f\left(k, \bar{x}_{k}\right), \quad k \geq k_{0}, \\
\bar{x}(k)=\left\{\begin{array}{l}
x\left(k_{m}\right)+I_{m}\left(k_{m}, x\left(k_{m}\right)\right), \quad k=k_{m}, \\
x(k), \quad k \neq k_{m}, m \in \mathbb{N},
\end{array}\right. \\
x(s)=\phi(s), \quad s \in \mathbb{N}_{k_{0}-\tau},
\end{array}\right.
$$

where $x(k) \in \mathbb{R}^{n}, \bar{x}_{k}$ is defined by $\bar{x}_{k}(s)=\bar{x}(k+s)$ for any $s \in \mathbb{N}_{-\tau}$. We assume $f: \mathbb{N} \times \mathbb{N}_{k_{0}-\tau} \rightarrow$ $\mathbb{R}^{n}$ and $I_{m}: \mathbb{N} \times \mathbb{R}^{n} \rightarrow \mathbb{R}^{n}$ for $m \in \mathbb{N}$, and the impulsive moments satisfy $0 \leq k_{0}<k_{1}<k_{2}<$ $\cdots, k_{m} \rightarrow \infty$ for $m \rightarrow \infty$. Furthermore, we assume $f(k, 0)=0, I_{m}(k, 0)=0$, which implies that $(2.1)$ admits the trivial solution. Let $x\left(k ; k_{0}, \phi\right)$ denote the trajectory of system (2.1) with initial condition $\phi$.

Definition $2.1([2,23])$ The trivial solution of system (2.1) is exponentially practically stable in the $p$ th moment, if, for any $k \geq k_{0}$ there exist constants $\lambda>0, M \geq 0, r>0$ such 
that

$$
\left\|x\left(k ; k_{0}, \phi\right)\right\|^{p} \leq M\|\phi\|^{p} e^{-\lambda\left(k-k_{0}\right)}+r .
$$

The following proposition will be used in the proof of our main results. The proofs are straightforward and will be omitted.

Proposition 2.1 Let $\gamma, \beta$ be positive real numbers and $0<\lambda<1$. If one of the following conditions holds:

(i) $\gamma \geq 1$ and $\frac{\gamma e-1}{\gamma e}<\beta<1$,

(ii) $\gamma<1$ and $\frac{e-1}{e}<\beta<1$,

then $\max \left\{(1-\beta) e^{\lambda},(1-\beta) \gamma e^{\lambda}\right\}<1$.

\section{Main results}

We first consider the exponentially practical stability problem of system (2.1) in the case $\gamma \geq 1$.

Theorem 3.1 If there exist positive numbers $a, c_{1}, c_{2}, p, \gamma, q, \beta, \eta ; q>\gamma \geq 1, \frac{\gamma e-1}{\gamma e}<\beta<1, \frac{1}{\gamma}>$ $1-\beta, \eta<\min \left\{\frac{a-a \gamma+a \gamma \beta}{\gamma}, \beta a\right\}$ and a Lyapunov function $V(k, \bar{x}(k))$ such that the following conditions hold:

(i) $c_{1}\|\bar{x}(k)\|^{p} \leq V(k, \bar{x}(k)) \leq c_{2}\|\bar{x}(k)\|^{p}+a, \forall k \geq k_{0}-\tau, x \in \mathbb{R}^{n}$,

(ii) if $V(k+s, \bar{x}(k+s))<q V(k+1, x(k+1))$ with $s \in \mathbb{N}_{-\tau}$, then $\Delta V(k, \bar{x}(k))=V(k+1, x(k+1))-V(k, \bar{x}(k)) \leq-\beta V(k, \bar{x}(k))+\eta$ holds,

(iii) $V\left(k_{m}, \bar{x}\left(k_{m}\right)\right)=V\left(k_{m}, x\left(k_{m}\right)\right)+I_{m}\left(k_{m}, x\left(k_{m}\right)\right) \leq \gamma V\left(k_{m}, x\left(k_{m}\right)\right), m \in \mathbb{N}, x \in \mathbb{R}^{n}$,

then the trivial solution of system (2.1) is exponentially practically stable in the pth moment.

Proof Since $q>\gamma \geq 1$, there exists $0<\lambda<1$ such that

$$
q>\gamma e^{\lambda(\tau+1)} \geq e^{\lambda(\tau+1)} .
$$

From (i), for $k \in\left[k_{0}-\tau, k_{0}\right]$, we get

$$
V(k, x(k)) \leq c_{2}\|x\|^{p}+a \leq c_{2}\|x\|^{p} e^{-\lambda\left(k-k_{0}\right)}+a \leq c_{2}\|\phi\|^{p} e^{-\lambda\left(k-k_{0}\right)}+a .
$$

We claim that

$$
V(k, \bar{x}(k)) \leq c_{2}\|\phi\|^{p} e^{-\lambda\left(k-k_{0}\right)}+a, \quad k \in\left[k_{m-1}+1, k_{m}\right] .
$$

Now, we will prove (3.2) by using mathematical induction.

First, we show that (3.2) holds for $m=1$, namely

$$
V(k, \bar{x}(k)) \leq c_{2}\|\phi\|^{p} e^{-\lambda\left(k-k_{0}\right)}+a, \quad k \in\left[k_{0}+1, k_{1}\right] .
$$

We assume (3.3) were not true, then there exists $k \in\left[k_{0}+1, k_{1}\right]$ such that $V(k, \bar{x}(k))>$ $c_{2}\|\phi\|^{p} e^{-\lambda\left(k-k_{0}\right)}+a$. Let $k^{*}=\min \left\{k \in\left[k_{0}+1, k_{1}\right] / V(k, \bar{x}(k))>c_{2}\|\phi\|^{p} e^{-\lambda\left(k-k_{0}\right)}+a\right\}$. From (3.1) and the definition of $k^{*}$, we have

$$
V(k, \bar{x}(k)) \leq c_{2}\|\phi\|^{p} e^{-\lambda\left(k-k_{0}\right)}+a, \quad k \in\left[k_{0}-\tau, k^{*}-1\right] .
$$


We will consider two possible cases for $k^{*} \in\left[k_{0}+1, k_{1}\right]$.

(I) $k^{*} \in\left[k_{0}+1, k_{1}-1\right]$.

In this case, for any $s \in \mathbb{N}_{-\tau}$, we have

$$
\begin{aligned}
V\left(k^{*}-1+s, x\left(k^{*}-1+s\right)\right) & \leq c_{2}\|\phi\|^{p} e^{-\lambda\left(k^{*}-1+s-k_{0}\right)}+a \\
& =c_{2}\|\phi\|^{p} e^{-\lambda(s-1)} e^{-\lambda\left(k^{*}-k_{0}\right)}+a \\
& \leq c_{2} e^{\lambda(\tau+1)}\|\phi\|^{p} e^{-\lambda\left(k^{*}-k_{0}\right)}+a e^{\lambda(\tau+1)} \\
& =e^{\lambda(\tau+1)}\left[c_{2}\|\phi\|^{p} e^{-\lambda\left(k^{*}-k_{0}\right)}+a\right] \\
& <q V\left(k^{*}, x\left(k^{*}\right)\right) .
\end{aligned}
$$

Let $\bar{k}=k^{*}-1$, then we get $V(\bar{k}+s, x(\bar{k}+s)) \leq q V(\bar{k}+1, x(\bar{k}+1))$. By (ii), we have $\Delta V(\bar{k}, x(\bar{k})) \leq-\beta V(\bar{k}, x(\bar{k}))+\eta$ holds. Thus, we have

$$
\begin{aligned}
V\left(k^{*}, x\left(k^{*}\right)\right) & \leq(1-\beta) V\left(k^{*}-1, x\left(k^{*}-1\right)\right)+\eta \\
& \leq(1-\beta)\left[c_{2}\|\phi\|^{p} e^{-\lambda\left(k^{*}-1-k_{0}\right)}+a\right]+\eta \\
& \leq(1-\beta) e^{\lambda} c_{2}\|\phi\|^{p} e^{-\lambda\left(k^{*}-k_{0}\right)}+a-\beta a+\eta .
\end{aligned}
$$

By assumption of $\eta$ and Proposition 2.1, we get $V\left(k^{*}, x\left(k^{*}\right)\right) \leq(1-\beta) e^{\lambda} c_{2}\|\phi\|^{p} e^{-\lambda\left(k^{*}-k_{0}\right)}+$ $a-\beta a+\eta$, which contradicts the definition of $k^{*}$. Hence (3.3) holds.

(II) $k^{*}=k_{1}$.

In this case, we have $V\left(k_{1}, \bar{x}\left(k_{1}\right)\right)>c_{2}\|\phi\|^{p} e^{-\lambda\left(k_{1}-k_{0}\right)}+a$ and for any $s \in \mathbb{N}_{-\tau}$, we get

$$
\begin{aligned}
V\left(k_{1}-1+s, x\left(k_{1}-1+s\right)\right) & \leq c_{2}\|\phi\|^{p} e^{-\lambda\left(k_{1}-1+s-k_{0}\right)}+a \\
& =c_{2}\|\phi\|^{p} e^{-\lambda(s-1)} e^{-\lambda\left(k_{1}-k_{0}\right)}+a \\
& \leq c_{2} e^{\lambda(\tau+1)}\|\phi\|^{p} e^{-\lambda\left(k_{1}-k_{0}\right)}+a e^{\lambda(\tau+1)} \\
& =e^{\lambda(\tau+1)}\left[c_{2}\|\phi\|^{p} e^{-\lambda\left(k_{1}-k_{0}\right)}+a\right] \\
& <e^{\lambda(\tau+1)} V\left(k_{1}, \bar{x}\left(k_{1}\right)\right) \\
& <e^{\lambda(\tau+1)} \gamma V\left(k_{1}, x\left(k_{1}\right)\right) \\
& <q V\left(k_{1}, x\left(k_{1}\right)\right) .
\end{aligned}
$$

Let $\bar{k}=k_{1}-1$, then we get $V(\bar{k}+s, x(\bar{k}+s)) \leq q V(\bar{k}+1, x(\bar{k}+1))$. By (ii), we have $\Delta V(\bar{k}, x(\bar{k})) \leq$ $-\beta V(\bar{k}, x(\bar{k}))+\eta$, which gives

$$
\frac{1}{\gamma} V\left(k_{1}, \bar{x}\left(k_{1}\right)\right) \leq V\left(k_{1}, x\left(k_{1}\right)\right) \leq(1-\beta) V\left(k_{1}-1, x\left(k_{1}-1\right)\right)+\eta .
$$

Thus, we obtain

$$
\begin{aligned}
V\left(k_{1}, \bar{x}\left(k_{1}\right)\right) & \leq(1-\beta) \gamma V\left(k_{1}-1, x\left(k_{1}-1\right)\right)+\eta \gamma \\
& \leq(1-\beta) \gamma\left[c_{2}\|\phi\|^{p} e^{-\lambda\left(k_{1}-1-k_{0}\right)}+a\right]+\eta \gamma \\
& \leq(1-\beta) \gamma e^{\lambda} c_{2}\|\phi\|^{p} e^{-\lambda\left(k_{1}-k_{0}\right)}+a \gamma-a \gamma \beta+\eta \gamma .
\end{aligned}
$$


By assumption of $\eta$ and Proposition 2.1, we get $V\left(k^{*}, x\left(k^{*}\right)\right) \leq(1-\beta) \gamma e^{\lambda} c_{2}\|\phi\|^{p} e^{-\lambda\left(k^{*}-k_{0}\right)}+$ $a \gamma-a \gamma \beta+\eta \gamma$, which contradicts the definition of $k^{*}=k_{1}$. Hence (3.3) holds.

Next, we assume (3.2) holds for $m \in \mathbb{N}$, namely

$$
V(k, \bar{x}(k)) \leq c_{2}\|\phi\|^{p} e^{-\lambda\left(k-k_{0}\right)}+a, \quad k \in\left[k_{m-1}+1, k_{m}\right]
$$

Then we will show that

$$
V(k, \bar{x}(k)) \leq c_{2}\|\phi\|^{p} e^{-\lambda\left(k-k_{0}\right)}+a, \quad k \in\left[k_{m}+1, k_{m+1}\right]
$$

We assume (3.4) were not true, then there exists

$$
k^{*}=\min \left\{k \in\left[k_{m}+1, k_{m+1}\right] / V(k, \bar{x}(k))>c_{2}\|\phi\|^{p} e^{-\lambda\left(k-k_{0}\right)}+a\right\} .
$$

For $k \in\left[k_{m}+1, k^{*}-1\right]$, we have $V(k, \bar{x}(k)) \leq c_{2}\|\phi\|^{p} e^{-\lambda\left(k-k_{0}\right)}+a$. We will consider two possible cases for $k^{*} \in\left[k_{m}+1, k_{m+1}\right]$.

(I) $k^{*} \in\left[k_{m}+1, k_{m+1}-1\right]$.

In this case, for any $s \in \mathbb{N}_{-\tau}$, we have

$$
\begin{aligned}
V\left(k^{*}-1+s, x\left(k^{*}-1+s\right)\right) & \leq c_{2}\|\phi\|^{p} e^{-\lambda\left(k^{*}-1+s-k_{0}\right)}+a \\
& =c_{2}\|\phi\|^{p} e^{-\lambda(s-1)} e^{-\lambda\left(k^{*}-k_{0}\right)}+a \\
& \leq c_{2} e^{\lambda(\tau+1)}\|\phi\|^{p} e^{-\lambda\left(k^{*}-k_{0}\right)}+a e^{\lambda(\tau+1)} \\
& =e^{\lambda(\tau+1)}\left[c_{2}\|\phi\|^{p} e^{-\lambda\left(k^{*}-k_{0}\right)}+a\right] \\
& <q V\left(k^{*}, x\left(k^{*}\right)\right),
\end{aligned}
$$

thus $V(\bar{k}+s, x(\bar{k}+s)) \leq q V(\bar{k}+1, x(\bar{k}+1))$ and from (ii), we have $\Delta V(\bar{k}, x(\bar{k})) \leq-\beta V(\bar{k}$, $x(\bar{k}))+\eta$, which contradicts the definition of $k^{*}$. Hence (3.4) holds.

(II) $k^{*}=k_{m+1}$.

In this case, we have $V\left(k_{m+1}, \bar{x}\left(k_{m+1}\right)\right)>c_{2}\|\phi\|^{p} e^{-\lambda\left(k_{m+1}-k_{0}\right)}+a$. Then, for any $s \in \mathbb{N}_{-\tau}$, we get

$$
\begin{aligned}
V\left(k_{m+1}-1+s, \bar{x}\left(k_{m+1}-1+s\right)\right) & \leq c_{2}\|\phi\|^{p} e^{-\lambda\left(k_{m+1}-1+s-k_{0}\right)}+a \\
& =c_{2}\|\phi\|^{p} e^{-\lambda(s-1)} e^{-\lambda\left(k_{m+1}-k_{0}\right)}+a \\
& \leq c_{2} e^{\lambda(\tau+1)}\|\phi\|^{p} e^{-\lambda\left(k_{m+1}-k_{0}\right)}+a e^{\lambda(\tau+1)} \\
& =e^{\lambda(\tau+1)}\left[c_{2}\|\phi\|^{p} e^{-\lambda\left(k_{m+1}-k_{0}\right)}+a\right] \\
& <e^{\lambda(\tau+1)} V\left(k_{1}, \bar{x}\left(k_{m+1}\right)\right) \\
& <e^{\lambda(\tau+1)} \gamma V\left(k_{1}, x\left(k_{m+1}\right)\right) \\
& <q V\left(k_{1}, x\left(k_{m+1}\right)\right) .
\end{aligned}
$$

Let $\bar{k}=k_{m+1}-1$, then we get $V(\bar{k}+s, \bar{x}(\bar{k}+s)) \leq q V(\bar{k}+1, x(\bar{k}+1))$. By (ii), we have $\Delta V(\bar{k}, \bar{x}(\bar{k})) \leq-\beta V(\bar{k}, \bar{x}(\bar{k}))+\eta$, which gives

$$
\frac{1}{\gamma} V\left(k_{m+1}, \bar{x}\left(k_{m+1}\right)\right) \leq V\left(k_{m+1}, x\left(k_{m+1}\right)\right) \leq(1-\beta) V\left(k_{m+1}-1, \bar{x}\left(k_{m+1}-1\right)\right)+\eta .
$$


Thus, we obtain

$$
\begin{aligned}
V\left(k_{m+1}, \bar{x}\left(k_{m+1}\right)\right) & \leq(1-\beta) \gamma V\left(k_{m+1}-1, \bar{x}\left(k_{m+1}-1\right)\right)+\eta \gamma \\
& \leq(1-\beta) \gamma\left[c_{2}\|\phi\|^{p} e^{-\lambda\left(k_{m+1}-1-k_{0}\right)}+a\right]+\eta \gamma \\
& \leq(1-\beta) \gamma e^{\lambda} c_{2}\|\phi\|^{p} e^{-\lambda\left(k_{m+1}-k_{0}\right)}+a \gamma-a \gamma \beta+\eta \gamma .
\end{aligned}
$$

By assumption on $\eta$ and Proposition 2.1, we get $V\left(k^{*}, x\left(k^{*}\right)\right) \leq(1-\beta) \gamma e^{\lambda} c_{2}\|\phi\|^{p} e^{-\lambda\left(k^{*}-k_{0}\right)}+$ $a \gamma-a \gamma \beta+\eta \gamma$, which contradicts the definition of $k^{*}=k_{m+1}$. Hence (3.4) holds.

Thus, for all $k \geq k_{0}$, we have

$$
V(k, \bar{x}(k)) \leq c_{2}\|\phi\|^{p} e^{-\lambda\left(k-k_{0}\right)}+a
$$

From (i), we obtain

$$
c_{1}\|\bar{x}(k)\|^{p} \leq V(k, \bar{x}(k)) \leq c_{2}\|\phi\|^{p} e^{-\lambda\left(k-k_{0}\right)}+a,
$$

which implies that

$$
\|\bar{x}(k)\|^{p} \leq \frac{c_{2}}{c_{1}}\|\phi\|^{p} e^{-\lambda\left(k-k_{0}\right)}+\frac{a}{c_{1}} .
$$

Therefore, the trivial solution of system (2.1) is exponentially practically stable in the $p$ th moment.

Next, we give an exponentially practical stability condition of system (2.1) for the case $0<\gamma<1$.

Theorem 3.2 If there exist positive numbers $a, c_{1}, c_{2}, p, \gamma, q, \beta, \eta ; q>1>\gamma>0, \frac{e-1}{e}<\beta<$ $1, \frac{1}{\gamma}>1-\beta, \eta<\min \left\{\frac{a-a \gamma+a \gamma \beta}{\gamma}, \beta a\right\}$ and a Lyapunov function $V(k, \bar{x}(k))$ such that the following conditions hold:

(i) $c_{1}\|\bar{x}(k)\|^{p} \leq V(k, \bar{x}(k)) \leq c_{2}\|\bar{x}(k)\|^{p}+a, \forall k \geq k_{0}-\tau, x \in \mathbb{R}^{n}$,

(ii) if $V(k+s, \bar{x}(k+s))<q V(k+1, x(k+1))$ with $s \in \mathbb{N}_{-\tau}$, then $\Delta V(k, \bar{x}(k))=V(k+1, x(k+1))-V(k, \bar{x}(k)) \leq-\beta V(k, \bar{x}(k))+\eta$ hold,

(iii) $V\left(k_{m}, \bar{x}\left(k_{m}\right)\right)=V\left(k_{m}, x\left(k_{m}\right)\right)+I_{m}\left(k_{m}, x\left(k_{m}\right)\right) \leq \gamma V\left(k_{m}, x\left(k_{m}\right)\right), m \in \mathbb{N}, x \in \mathbb{R}^{n}$, then the trivial solution of system (2.1) is exponentially practically stable in the pth moment.

Proof Since $q>1>\gamma>0$, there exists $0<\lambda<1$ such that

$$
q>e^{\lambda(\tau+1)} \geq \gamma e^{\lambda(\tau+1)} .
$$

From assumption of $\eta$ and Proposition 2.1, by using a similar argument as in the proof of Theorem 3.1, we may show that $\|\bar{x}(k)\|^{p} \leq \frac{c_{2}}{c_{1}}\|\phi\|^{p} e^{-\lambda\left(k-k_{0}\right)}+\frac{a}{c_{1}}, \forall k \geq k_{0}$. Therefore, the trivial solution of system (2.1) is exponentially practically stable in the $p$ th moment.

Remark 3.1 From the methods of proof of Theorem 3.1 and Theorem 3.2, it is clear that these methods can be applied for an impulsive discrete time system with time varying delay $\tau(k)$ with $0 \leq \tau(k) \leq \tau, \tau>0$. 
Remark 3.2 We now provide a direct and effective solving algorithm corresponding to Theorems 3.1 and 3.2 as follows:

1. First, we choose an appropriate Lyapunov function candidate. Then we make an estimate for $c_{1}, c_{2}$ and $a$ satisfying condition (i).

2. Next, find an estimation of $\gamma$ which satisfies (iii).

3. Finally, with estimates of $c_{1}, c_{2}, a$ and $\gamma$ in 1 and 2 , we choose appropriate $q, \beta$, and $\eta$ which satisfy condition (ii).

\section{Numerical example}

Example 4.1 Consider the following impulsive discrete time system with delay:

$$
\left\{\begin{array}{l}
x(k+1)=c x(k)+d x(k-1), \quad k \geq 0, \\
\bar{x}(k)=\left\{\begin{array}{l}
\gamma x\left(k_{m}\right), \quad k=k_{m}, \\
x(k), \quad k \neq k_{m}, m \in \mathbb{N},
\end{array}\right. \\
x(s)=\phi(s), \quad s \in \mathbb{N}_{-1},
\end{array}\right.
$$

where $c, d$ are arbitrary constants and $\gamma$ is positive constant, which is considered in [4]. Let $a>0$ be given. If there exist positive numbers $c_{1}, c_{2}, p, \gamma, q, \beta, \eta$ such that $\eta=(1-|c|-$ $|d|) a, \beta \leq \min \left\{1-\frac{|c|}{1-|d| q}, \gamma-\frac{|c|}{1-|d| q}\right\}$, then the trivial solution of system (4.1) is exponentially practically stable in the $p$ th moment.

Proof We choose a Lyapunov function $V(k, \bar{x}(k))=|\bar{x}(k)|+a$; then we have

(i) $c_{1}\|\bar{x}(k)\| \leq V(k, \bar{x}(k))=|\bar{x}(k)|+a \leq c_{2}\|\bar{x}(k)\|+a, \forall k \geq k_{0}-1$.

(ii) Assume $V(k+s, \bar{x}(k+s))<q V(k+1, x(k+1))$ with $s \in \mathbb{N}_{-1}$, then we have

$$
\begin{aligned}
V(k+1, x(k+1)) & =|x(k+1)|+a \\
& =|c x(k)+d x(k-1)|+a \\
& =|c||x(k)|+|d||x(k-1)|+a \\
& =|c||x(k)|+|c| a-|c| a+|d||x(k-1)|+|d| a-|d| a+a \\
& =|c| V(k, x(k))+|d| V(k-1, x(k-1))+(1-|c|-|d|) a \\
& \leq|c| V(k, x(k))+|d| q V(k+1, x(k+1))+(1-|c|-|d|) a \\
& \leq \frac{|c|}{1-|d| q} V(k, x(k))+(1-|c|-|d|) a .
\end{aligned}
$$

We have two cases as follows.

Case I. $k \neq k_{m}$. In this case, we have

$$
\begin{aligned}
\Delta V(k, \bar{x}(k)) & =V(k+1, x(k+1))-V(k, \bar{x}(k)) \\
& =V(k+1, x(k+1))-V(k, x(k)) \\
& \leq \frac{|c|}{1-|d| q} V(k, x(k))+(1-|c|-|d|) a-V(k, x(k)) \\
& =\left(\frac{|c|}{1-|d| q}-1\right) V(k, x(k))+(1-|c|-|d|) a
\end{aligned}
$$




$$
\begin{aligned}
& =-\left(1-\frac{|c|}{1-|d| q}\right) V(k, x(k))+(1-|c|-|d|) a \\
& =-\left(1-\frac{|c|}{1-|d| q}\right) V(k, x(k))+\eta ; \quad \eta=(1-|c|-|d|) a .
\end{aligned}
$$

Case II. $k=k_{m}$. In this case, we have

$$
\begin{aligned}
\Delta V(k, \bar{x}(k)) & =V(k+1, x(k+1))-V(k, \bar{x}(k)) \\
& \leq \frac{|c|}{1-|d| q} V(k, x(k))+(1-|c|-|d|) a-\gamma V(k, x(k)) \\
& =\left(\frac{|c|}{1-|d| q}-\gamma\right) V(k, x(k))+(1-|c|-|d|) a \\
& =-\left(\gamma-\frac{|c|}{1-|d| q}\right) V(k, x(k))+(1-|c|-|d|) a \\
& =-\left(\gamma-\frac{|c|}{1-|d| q}\right) V(k, x(k))+\eta ; \quad \eta=(1-|c|-|d|) a .
\end{aligned}
$$

From the assumptions, we get

$$
\Delta V(k, \bar{x}(k)) \leq-\beta V(k, \bar{x}(k))+\eta
$$

(iii) For $k=k_{m}$, we have

$$
\begin{aligned}
V\left(k_{m}, \bar{x}\left(k_{m}\right)\right) & =\left|\bar{x}\left(k_{m}\right)\right|+a \\
& =\left|\gamma x\left(k_{m}\right)\right|+a \\
& =\gamma\left[\left|x\left(k_{m}\right)\right|+\frac{a}{\gamma}\right] \\
& \leq \gamma\left[\left|x\left(k_{m}\right)\right|+a\right] \\
& =\gamma V\left(k_{m}, x\left(k_{m}\right)\right) .
\end{aligned}
$$

Therefore, from Theorem 3.2, we conclude that the system (4.1) is exponentially practically stable in the $p$ th moment. For simulation purposes, we let $a=0.5,|c|=0.04,|d|=$ $0.43, \lambda=0.05, q=1.4, \gamma=0.9$. We choose the Lyapunov function $V(k, \bar{x}(k))=|\bar{x}(k)|+0.5$. Then Theorem 3.2 is satisfied with the parameters $c_{1}=c_{2}=1, a=0.5, p=1, \beta=0.8, \gamma=$ 0.9 , and $\eta=0.27$. Therefore, we have $\|\bar{x}(k)\| \leq 1.8 e^{-0.05 k}+0.5, \forall k \geq 0$. In Figure 1 and Figure 2, with initial conditions given by $x(-1)=1.8, x(0)=1.6$, the trajectories of solutions of (4.1) with impulsive moments $k_{m}=4 k+3, m \in \mathbb{N}$ in which $\sup _{m \in \mathbb{Z}^{+}}\left\{k_{m+1}-k_{m}\right\}=4<+\infty$ and $k_{m}=\{4,6,9,13,18,24, \ldots\}$ in which $\sup _{m \in \mathbb{Z}^{+}}\left\{k_{m+1}-k_{m}\right\}=\infty$, are shown, respectively. It is worth noting that, in [4], the asymptotically practically stable criterion requires $\tau \triangleq \sup _{m \in \mathbb{Z}^{+}}\left\{k_{m+1}-k_{m}\right\}<+\infty$. On the other hand, this restriction is not required in Theorem 3.2. Therefore, our result is less conservative than the result obtained in [4].

\section{Conclusion}

In this paper the exponentially practical stability is derived for an impulsive discrete time system with delay by using Lyapunov stability theory and a Razumikhin type technique. 

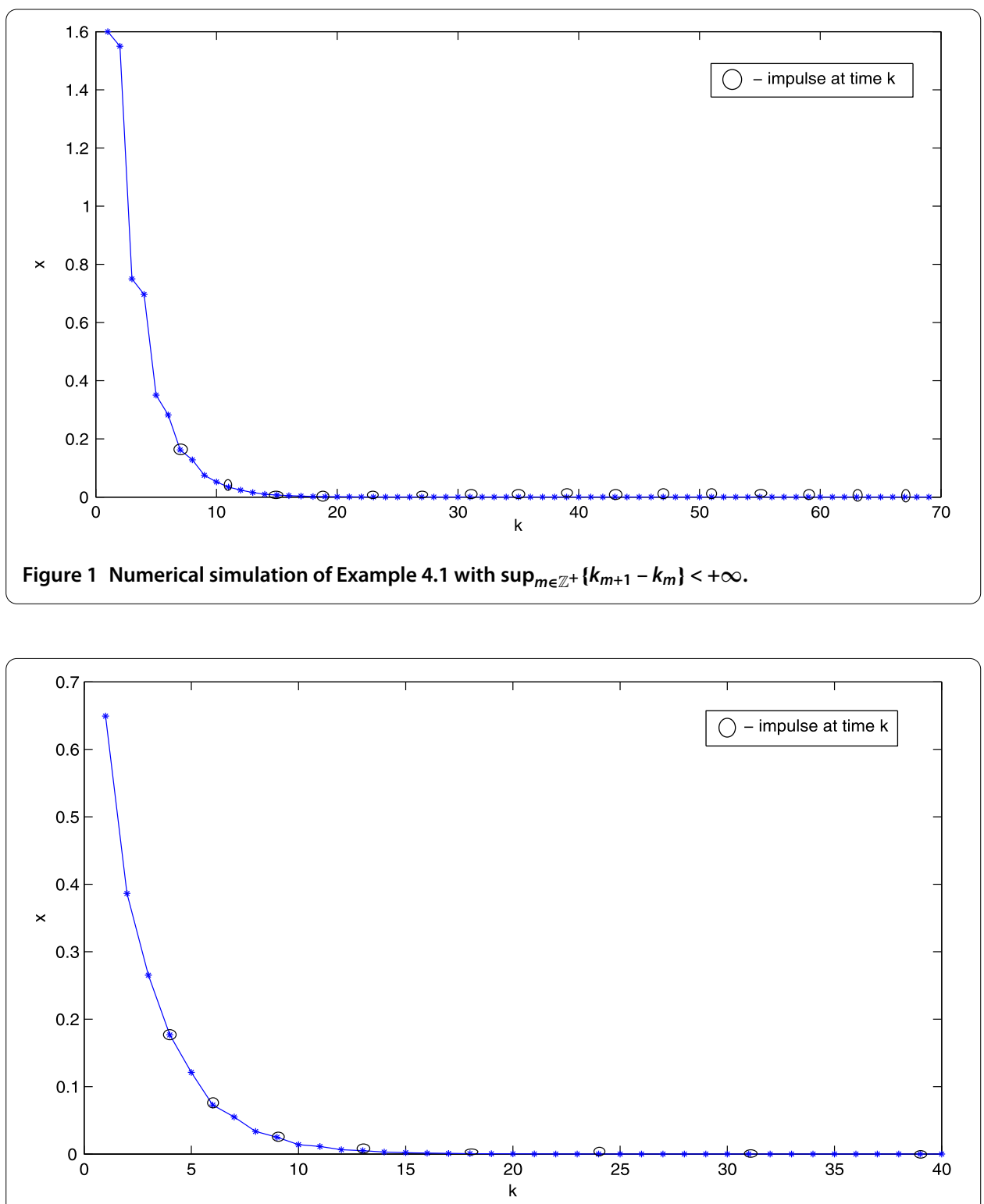

Figure 2 Numerical simulation of Example 4.1 with $\sup _{m \in \mathbb{Z}^{+}}\left\{k_{m+1}-k_{m}\right\}=\infty$.

Comparing to some existing results in the literature the obtained criterion is not required that $\sup _{m \in \mathbb{Z}^{+}}\left\{k_{m+1}-k_{m}\right\}<+\infty$, where $k_{m}$ are impulsive moments. A numerical example is given to show the effectiveness of our theoretical results.

Competing interests

The authors declare that they have no competing interests.

Authors' contributions

All authors have equal contributions to the writing of this paper. All authors have read and approved the final version of the manuscript.

\section{Acknowledgements}

The first author is supported by student scholarship from the Human Resources Development in Science Project (Science Achievement Scholarship of Thailand SAST) and Chiang Mai University. The second author is supported by Chiang Mai University. 
Received: 22 June 2016 Accepted: 18 October 2016 Published online: 28 October 2016

\section{References}

1. Liu, B, Marquez, H: Razumikhin-type stability theorems for discrete delay systems. Automatica 43(7), 1219-1225 (2007)

2. Li, C, Duan, S, Wu, S, Liao, X: Exponential stability of impulsive discrete systems with time delay and applications in stochastic neural networks: a Razumikhin approach. Neurocomputing 82, 29-36 (2012)

3. Wu, K, Ding, X: Impulsive stabilization of delay difference equation and its application in Nicholson's blowflies model. Adv. Differ. Equ. 2012, 88 (2012)

4. Zhang, K, Liu, X: Global exponential stability of nonlinear impulsive discrete systems with time delay. In: 2013 25th Chinese Control and Decision Conference (CCDC), pp. 148-153 (2013)

5. Sun, L, Liu, C, Li, X: Practical stability of impulsive discrete systems with time delays. Abstr. Appl. Anal. 2014, 954121 (2014)

6. Xiang, M, Xiang, Z: Exponential stability of discrete time switched linear positive systems with time delay. Appl. Math. Comput. 230, 193-199 (2014)

7. Zhang, Q, Liu, W, Su, Z: Practical stability and controllability for a class of nonlinear discrete systems with time delay. Nonlinear Dyn. Syst. Theory 10(2), 161-174 (2010)

8. Liu, X, Zhang, Z: Uniform asymptotic stability of impulsive discrete systems with time delay. Nonlinear Anal. 72, 4941-4950 (2011)

9. Zhang, Y, Sun, J, Feng, G: Impulsive control of discrete systems with time delay. IEEE Trans. Autom. Control 54, 830-834 (2009)

10. Ellouze, I, Hammami, MA: Practical stability of impulsive control systems with multiple time delays. Dyn. Contin. Discrete Impuls. Syst., Ser. A Math. Anal. 20, 341-356 (2013)

11. Yan, J, Shen, J: Impulsive stabilization of functional differential equations by Lyapunov-Razumikhin functions. Nonlinear Anal. 37, 245-255 (1999)

12. Dlala, M, Hammami, MA: Uniform exponential practical stability of impulsive perturbed systems. J. Dyn. Control Syst. 13, 373-386 (2007)

13. Wang, Q, Liu, X: Impulsive stabilization of delay differential systems via the Lyapunov-Razumikhin method. Appl. Math. Lett. 20, 839-845 (2007)

14. Peng, SG, Zhang, Y: Razumikhin-type theorems on $p$ th moment exponential stability of impulsive stochastic delayed differential equations. IEEE Trans. Autom. Control 55(8), 2795-2805 (2010)

15. Fu, X, Li, X: Razumikhin-type theorems on exponential stability of impulsive infinite delay differential systems. J. Comput. Appl. Math. 224, 1-10 (2009)

16. Hamed, BB, Ellouze, I, Hammami, MA: Practical uniform stability of nonlinear differential delay equations. Mediterr. J. Math. 8, 603-616 (2011)

17. Wu, B, Han, J, Cai, X: On the practical stability of impulsive differential equations with infinite delay in terms of two measures. Abstr. Appl. Anal. 2012, 434137 (2012)

18. Sun, G, Zhang, Y: Exponential stability of impulsive discrete-time stochastic BAM neural networks with time-varying delay. Neurocomputing 131, 323-330 (2014)

19. Huang, S, Li, X, Xiang, Z: Anti-windup design and I2-gain analysis for a class of discrete-time impulsive switched systems with actuator saturation. Trans. Inst. Meas. Control 38(4), 425-434 (2016)

20. Li, X, Xiang, Z: Observer design of discrete-time impulsive switched nonlinear systems with time-varying delays. Appl. Math. Comput. 229, 327-339 (2014)

21. Li, X, Xiang, Z, Karimi, HR: Asynchronously switched control of discrete impulsive switched systems with time delays. Inf. Sci. 249, 132-142 (2013)

22. Li, Z, Fang, J, Miao, Q, He, G: Exponential synchronization of impulsive discrete-time complex networks with time-varying delay. Neurocomputing 157, 335-343 (2015)

23. Ghanmi, B, Hammami, MA, Hadj Taied, N: Growth conditions for exponential stability of time-varying perturbed systems. Int. J. Control 89(6), 1086-1097 (2013)

24. Benabdallah, A, Ellouze, I, Hammami, MA: Practical stability of nonlinear time-varying cascade systems. J. Dyn. Control Syst. 15, 45-62 (2009)

25. Song, X, Li, S, Li, A: Practical stability of nonlinear differential equation with initial time difference. Appl. Math. Comput. 203, 157-162 (2008) 